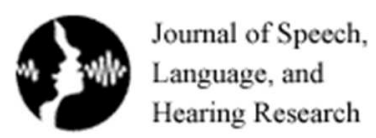

\title{
Non-Adjacent Dependency Learning in Cantonese-Speaking Children With and Without a History of Specific Language Impairment
}

\begin{tabular}{|r|l|}
\hline Journal: & Journal of Speech, Language, and Hearing Research \\
\hline Manuscript ID & JSLHR-L-15-0232.R4 \\
\hline Manuscript Type: & Research Note \\
\hline Date Submitted by the Author: & n/a \\
\hline Complete List of Authors: & $\begin{array}{l}\text { Iao, Lai-Sang; Nottingham Trent University, Division of Psychology } \\
\text { Ng, Lai Yan; University of Hong Kong, Division of Speech and Hearing } \\
\text { Sciences } \\
\text { Wong, Anita; University of Hong Kong, Division of Speech and Hearing } \\
\text { Sciences } \\
\text { Lee, Oi Ting; University of Hong Kong, Division of Speech and Hearing } \\
\text { Sciences }\end{array}$ \\
\hline Keywords: & \begin{tabular}{l} 
Children, Cognition, Language, Specific language impairment, Development \\
\hline
\end{tabular} \\
\hline
\end{tabular}


Running head: NON-ADJACENT DEPENDENCY LEARNING IN SLI

Non-Adjacent Dependency Learning in Cantonese-Speaking Children With and Without a History of Specific Language Impairment

Lai-Sang Iao ${ }^{\mathrm{a}, \mathrm{b}, \mathrm{c}^{*}}$, Lai Yan $\mathrm{Ng}^{\mathrm{b}}$, Anita Mei Yin Wong ${ }^{\mathrm{a}, \mathrm{b}}$ and Oi Ting Lee ${ }^{\mathrm{b}}$

${ }^{\mathrm{a} C e n t r e ~ f o r ~ C o m m u n i c a t i o n ~ D i s o r d e r s, ~ U n i v e r s i t y ~ o f ~ H o n g ~ K o n g, ~ 5 / F, ~ P r i n c e ~ P h i l i p ~}$ Dental Hospital, 34 Hospital Road, Sai Ying Pun, Hong Kong

${ }^{b}$ Division of Speech and Hearing Sciences, University of Hong Kong, 7/F Meng Wah Complex, University of Hong Kong, Pokfulam, Hong Kong

${ }^{\mathrm{c}}$ Division of Psychology, Nottingham Trent University, Burton Street, Nottingham, United Kingdom NG1 4BU

${ }^{*}$ Corresponding author

Address for correspondence: Division of Psychology, Nottingham Trent University, Burton Street, Nottingham, United Kingdom NG1 4BU

E-mail: lai-sang.iao@,ntu.ac.uk

Phone: +44 (0)115 8482387 


\begin{abstract}
Purpose: This study investigated non-adjacent dependency learning in Cantonese-speaking children with and without a history of Specific Language Impairment (SLI) in an artificial linguistic context.

Method: Sixteen Cantonese-speaking children with SLI history and 16 Cantonese-speaking children with typical language development (TLD) were tested with a non-adjacent dependency learning task using artificial languages that mimic Cantonese.

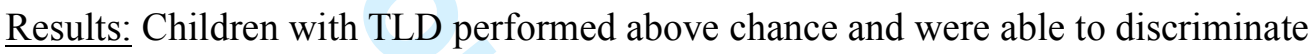
between trained and untrained non-adjacent dependencies. However, children with SLI history performed at chance and were not able to differentiate trained versus untrained non-adjacent dependencies.

Conclusions: These findings, together with previous findings with English-speaking adults and adolescents with language impairments, suggested that individuals with atypical language development, regardless of age, diagnostic status, language and culture, showed difficulties in learning non-adjacent dependencies. This study provides evidence for early impairments to statistical learning in individuals with atypical language development.
\end{abstract}

Keywords: Non-adjacent dependency learning, Statistical learning, Language acquisition, Specific Language Impairment 
Non-Adjacent Dependency Learning in Cantonese-Speaking Children With and Without a History of Specific Language Impairment

Language consists of rules and regularities that embed in a continuous sequence. Extracting these rules and regularities and using them productively are essential for language acquisition and social communication. Some of these rules and regularities occur among adjacent elements (e.g., bound-morpheme such as $-s$ is added to unbound-morphemes such as $d o g$ to illustrate the plural of $d o g$ ) while others exhibit non-adjacent dependencies (e.g., morphosyntactic rules such as is X-ing where the intervening $\mathrm{X}$ is a verb). Much research has been devoted to the investigation of how English speakers acquire these adjacent and non-adjacent dependencies and a domain-general statistical learning mechanism was suggested to explain the acquisition (e.g., Gómez, 2002; Saffran, Aslin, \& Newport, 1996). However, there is no study investigating the acquisition of linguistic dependencies in non-inflectional languages such as Cantonese which is a Chinese dialect predominantly used as a native language in Hong Kong, Macau, Guangzhou, and many Chinese communities in other countries. Moreover, it is found that the features of Cantonese may affect the profile of linguistic deficits in certain types of language disorders, e.g., Specific Language Impairment (SLI; Leonard, 1998). Given that English speakers with SLI were found to have difficulties in learning non-adjacent dependencies (Hsu, Tomblin, \& Christiansen, 2014), the current study sought to investigate whether Cantonese-speaking children with a history of SLI would also show these difficulties when comparing to Cantonese-speaking children with typical language development (TLD).

In non-adjacent dependencies, there is usually a considerable variation in intervening elements (e.g., the verb in progressives and perfectives in English, such as is go-ing, is draw-ing, has jump-ed, has kick-ed). Therefore, a language learner has to notice the reoccurring dependencies that surround the variations in order to acquire the 
dependencies in a particular language. Gómez (2002) was the first to investigate the learning of non-adjacent dependencies in typically developing infants and adults. In this study, participants were exposed to one of two artificial languages that mimic English. Each of the two languages involved strings of three elements (e.g., pel wadim jic or vot kicey rud). The strings began and ended with the same elements (e.g., beginning elements: pel and vot; ending elements: jic and $r u d$ ) but the two languages differed in the dependencies between the beginning and ending elements (e.g., Language 1: pel paired with jic, vot paired with rud; Language 2: pel paired with rud, vot paired with jic). In other words, if a participant was exposed to Language $1, \mathrm{~s} /$ he was trained on the dependencies of pel - jic and vot - rud. The intervening elements (e.g., wadim and kicey) varied within the dependencies and the same set of intervening elements appeared in both languages. As a result, participants could only discriminate the two languages in a later test by noticing the difference in the non-adjacent dependencies between the beginning and ending elements.

Gómez (2002) found that a large set size of 24 intervening elements resulted in excellent discrimination between trained and untrained non-adjacent dependencies. She explained this finding in terms of statistical learning. When the set size of the intervening elements was small, the beginning elements were followed by a few possible intervening elements so the statistical relationship between these adjacent elements was too easy to notice and participants missed the non-adjacent dependencies between the beginning and ending elements. In contrast, when the intervening elements were highly variable, the beginning elements hardly predicted the intervening elements so the non-adjacent dependencies between the beginning and ending elements became the only reliable statistical relationship between the elements. Since then, statistical learning has been used as the main explanation for non-adjacent dependency learning (e.g., Conway \& Christiansen, 2006; Gebhart, Newport, \& Aslin, 2009; Newport \& Aslin, 2004). 
Further studies also applied Gómez's (2002) paradigm to test individuals with language disorders, including adults with language-based learning difficulties (LLD; Grunow, Spaulding, Gómez, \& Plante, 2006) and adolescents with SLI (Hsu et al., 2014). Both studies showed that individuals with language disorders could not discriminate the trained and untrained non-adjacent dependencies despite a large set size of 24 intervening elements being used. Grunow et al. also tested whether participants were able to generalize the trained non-adjacent dependencies to strings that involved novel intervening elements. Results showed that adults without LLD were able to generalize when the set size of intervening elements was 24 but adults with LLD could not. However, Plante et al. (2014) trained children with language impairment on adjacent and non-adjacent dependencies and generalization was found when training involved variability as high as 24 exemplars. Hence, it is not clear whether impairments to statistical learning underlie individuals with language disorders' difficulties in learning and generalizing non-adjacent dependencies. One possible way to explain this discrepancy in the literature is that Grunow et al. and Hsu et al. tested artificial language learning in an experimental context whereas Plante et al. tested natural language learning in a treatment context. Another possibility is that adults with LLD and adolescents with SLI have been having language disorders for years and that may have contributed to further difficulties in learning and generalizing non-adjacent dependencies. In order to address this discrepancy in the literature, the current study tested children with a history of SLI in similar experimental context. If difficulties in learning and generalizing non-adjacent dependencies were also found in this study, this would suggest that these difficulties may be explained by early impairments to statistical learning rather than long years of language impairments.

In Cantonese, non-adjacent dependencies can be seen in the use of temporal 
adverbs when followed by monosyllabic verbs (e.g., 曾經 食 過/cang4 ging 1 sik6 gwo3/ which means ' has eaten'; Ding, 2008) and in anaphoric reference (e.g., 小明打 緊波。侣 好開心。/siu2 ming4 daa2 gan2 bo1. keoi5 ho2 hoi1 sum./ which means 'Siu-Ming is playing a ball. $\underline{\mathrm{He}}$ is very happy.'). Other Chinese languages such as Mandarin have similar non-adjacent dependency examples. As there is an increasing number of people learning and using Chinese across the world, it is important to examine non-adjacent dependency learning in an artificial language that mimics Chinese which is a non-inflectional language and has not been investigated yet. Given that this was the first study that investigated non-adjacent dependency learning in Cantonese-speaking children, Gómez’s (2002) design was adopted to develop two artificial languages that mimic Cantonese and involve non-adjacent dependencies. It was expected that Cantonese-speaking children with TLD would learn the non-adjacent dependencies whereas those with SLI history would not. If children with SLI history did not learn the non-adjacent dependencies in this experimental context, it was likely that they would not show generalization either. Ethical approval was obtained from the ethics committee in the University of Hong Kong and parental informed consent was obtained before testing.

\section{Methods}

\section{Participants}

Thirty-two children who had completed a three-year longitudinal project on reading development were recruited. Figure 1 outlines the recruitment procedure. All children with SLI scored more than 1.25 SD below the mean for age on at least two of the six subtests in the Hong Kong Cantonese Oral Language Assessment Scale (HKCOLAS; T'sou et al., 2006) in Year 1 and 2 of the project, despite the absence of cognitive, peripheral hearing, psychosocial or neurological impairments. All children scored above the cut-off of 85 on the Raven's Standard Progressive Matrices (SPM; Raven, 1986) in Year 1. They were also tested with the Hong Kong Test of Specific Learning Difficulties 
in Reading and Writing for Primary School Students-Second Edition (HKT-P [II]; Ho, Chan, Chung, Tsang, Lee \& Cheng, 2007) to rule out dyslexia in Year 2. The HKT-P [II] comprises the literacy composite, phonological awareness composite, phonological memory composite, rapid automatic naming and orthographic skills composite. Dyslexia was defined as a scaled score of 7 or lower in the literacy composite and in one of the cognitive composites (Chan, Ho, Tsang, Lee, \& Chung, 2007). All children did not fit with this dyslexia definition. In Year 3, they received an assessment on reading comprehension and the current non-adjacent dependency learning task. Although children's current SLI status was not assessed, it was certain that children in the TD group had TLD whereas children in the SLI-only group had a history of SLI. Participant characteristics are presented in Table 1. The two groups did not differ in chronological age, $t(20.24)=1.26, p=.22$, and non-verbal intelligence based on earlier Raven's SPM scores, $t(30)=.09, p=.93$. All the other language measures obtained earlier were significantly different between the groups.

\section{------ Insert Figure 1 and Table 1------}

\section{Materials and procedure}

Adopting Gómez’s (2002) design, children listen to one of two artificial languages that mimic Cantonese. Simple randomization was used to randomly assign children to one of the two languages. The two languages involved strings of three elements and were equivalent in all aspects except the dependencies between the beginning and ending elements. These elements were syllables that have been checked with a database (Humanities Computing and Methodology Programme, 2003) to ensure non-existence in Cantonese (i.e., pseudomorphemes). Besides, Cantonese is not only a non-inflectional language but also a tone language that uses lexical tones to signify meanings. In terms of fundamental frequency patterning, the six contrastive tones in Cantonese can be described as Tone 1 (high level), Tone 2 (high rising), Tone 3 (mid level), Tone 4 (low 
falling), Tone 5 (low rising), and Tone 6 (low level). Ching (1981) and Wong, Ciocca, and Yung (2009) reported that Tone 1 and 4 were acoustically most salient and best identified even in difficult listening conditions. Thus, pseudomorphemes in Tone 1 and 4 were used (see Appendix A). There were 24 intervening pseudomorphemes in the training phase. In total, 48 strings ( 2 dependencies $\times 24$ intervening elements $)$ were generated for the training phase in each of two languages. All strings were recorded by a native Cantonese-speaking female with an inter-string pause of $750 \mathrm{~ms}$ and an inter-syllabic pause of $250 \mathrm{~ms}$. After piloting, each string was played four times at a random order to each child using the E-prime software via headphones. Volume was set at a comfortable listening level.

Children were required to listen carefully to strings of syllables that they have never heard before for about 10 minutes and to complete a test later. After training, children were informed that the strings they heard were generated with accord to a set of rules and that they were required to discriminate whether the strings at test followed the rules by pressing a 'yes' or 'no' button. In total, 24 strings were tested: 6 strings with trained intervening elements were taken from each of the two languages and 6 strings with 3 novel intervening elements were generated from each language for testing generalization (see Appendix B). Thus, if children have learnt the non-adjacent dependencies in the trained language, they should endorse more strings that involved the trained non-adjacent dependencies than those that did not.

\section{Results}

No significant difference was found between Language 1 and 2 so data for the two languages were averaged in the following analyses. Effect sizes were calculated using Cohen's $d$ and partial eta-squared $\left(\eta_{p}{ }^{2}\right)$. In general, an effect size of .20 is a small effect, .50 is a medium effect and .80 is a large effect (Cohen, 1992). Children's performance based on accuracy was first examined against chance level (i.e., .50) using a 
one-sampled $t$-test. Children with TLD performed above chance on the test items $(\operatorname{Min}-\operatorname{Max}=.50-1, M=.60, S D=.13), t(15)=3.18, p<.01, d=.80$, but not on the generalization items $($ Min-Max $=.33-.92, M=.56, S D=.15), t(15)=1.69, p=.11, d$ $=.42$. Children with SLI history performed at chance on both test (Min-Max $=.25-.75$, $M=.49, S D=.15)$ and generalization items $($ Min-Max $=.33-.75, M=.51, S D=.12)$, $t \mathrm{~s}(15)<.34, p \mathrm{~s}>.74$. D-prime $\left(d^{\prime}\right)$ was also calculated on the test and generalization items for each group. Based on signal detection analysis (Green \& Swets, 1966/1974), the parameter $d$ ' is distributed around 0 and a value of 0 means no discrimination between items. Children with TLD obtained a large $d$ ', indicating that they were able to correctly discriminate between the trained and untrained test items (Min-Max $=0-8.60, M=1.14$, $S D=2.21)$, however, their discrimination between the trained and untrained generalization items was less successful (Min-Max $=-.97-5.27, M=.73, S D=1.69)$. By contrast, children with SLI history obtained a small $d$ ' for both test items (Min-Max = $-4.30-4.30, M=-.40, S D=2.09)$ and generalization items $($ Min-Max $=-3.87-4.30, M$ $=.05, S D=1.58)$, suggesting that they were not able to discriminate between the trained and untrained items.

Given that literature in this area has compared correct accepts against false positives (Gómez, 2002; Grunow et al., 2006; Hsu et al., 2014), the same analysis was carried out to allow comparison between studies. Figure 2 presents the mean scores for correct accepts and false positives for test and generalization items in each group. To support the hypothesis that children with TLD would learn the non-adjacent dependencies whereas those with SLI history would not, the correct accepts should be significantly more than the false positives in the TLD group but not the SLI-history group for both test and generalization items. A 2x2x2 3-way mixed ANOVA with group as between-subjects variable and grammaticality (correct accepts vs. false positives) and item type (test items vs. generalization items) as within-subjects variables resulted in a 
main effect of grammaticality, $F(1,30)=4.71, p<.05, \eta_{p}{ }^{2}=.14$, and a main effect of item type, $F(1,30)=40.94, p<.001, \eta_{p}{ }^{2}=.58$, but more importantly, a significant grammaticality $\mathrm{x}$ group interaction, $F(1,30)=4.15, p=.05, \eta_{p}{ }^{2}=.12$. There were no other main effects or interactions, $F_{\mathrm{s}}<1.05, p \mathrm{~s}>.31, \eta_{p}{ }^{2} \mathrm{~s}<.03$. Post hoc tests further indicated that the grammaticality effect was significant for children with $\operatorname{TLD}(p<.01, d$ $=1.49)$ but not for children with SLI $(p=.92, d=.05)$, suggesting that the correct accepts were significantly more than the false positives in the TLD group but not the SLI-history group. Furthermore, the item type effect suggested that performance on test items was significantly better than for generalization items.

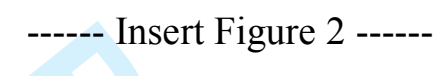

\section{Discussion}

This was the first study that investigated non-adjacent dependency learning in Cantonese-speaking children with and without SLI history in an artificial linguistic context. Although the task appeared to be difficult given children's accuracy was not high, children with TLD did perform above chance on the test items and were able to differentiate trained versus untrained non-adjacent dependencies. However, those children with SLI history performed at chance and were not able to differentiate trained versus untrained non-adjacent dependencies. These findings were consistent with those found in Grunow et al. (2006) and Hsu et al. (2014) although they tested adults with LLD and adolescents with SLI respectively. Therefore, individuals with atypical language development, regardless of age, diagnostic status, language and culture, showed difficulties in learning non-adjacent dependencies in artificial languages. This study thus provided stronger evidence for early impairments to statistical learning rather than long years of language impairments in explaining non-adjacent dependency learning in individuals with atypical language development.

However, the current study was not a longitudinal study and the sample size was 
small. Further longitudinal investigation with a much larger sample is needed to clarify whether early impairments to statistical learning has an impact on non-adjacent dependency learning and language acquisition. Currently, there is no longitudinal data available in either typical or atypical developing populations (Arciuli \& von Koss Torkildsen, 2012). Although Kerkhoff, De Bree, De Klerk, and Wijnen (2013) found an early difference between infants at familial risk of dyslexia and typically developing infants in non-adjacent dependency learning, there was no longitudinal follow-up on the infants' language development. Besides, this early difference in non-adjacent dependency learning between the two groups of infants may be due to attention during the training phrase. They indicated that the familial risk infants showed shorter looking times and higher degree of restlessness during the training phrase. Given that attention could be a factor affecting non-adjacent dependency learning and evidence has been found in adults (Pacton \& Perruchet, 2008), attention may explain the current findings in both children with and without SLI history. Although no obvious inattentive behaviour was observed during testing, attentive listening could not be assured. Children with SLI history could fail to learn any non-adjacent dependencies due to lack of attention whereas children with TLD may attend well to the dependencies in relation to the intervening elements and thus performed better on the test items than the generalisation items. There is also evidence showing that children with SLI have deficits in auditory attention (Finneran, Francis, \& Leonard, 2009; Spaulding, Plante, \& Vance, 2008). Future research may therefore investigate to what extent attention may affect children's non-adjacent dependency learning and whether attention may be better than statistical learning in explaining language impairments.

In addition to attention, exposure duration could be another factor that affects non-adjacent dependency learning. Although this effect was not found in adults (Romberg \& Saffran, 2013), it was found in children with SLI who showed sensitivity to transitional 
probabilities when the exposure duration was doubled (Evans, Saffran \& Robe-Torres, 2009). It is therefore possible that children with SLI history in the current study might learn the non-adjacent dependencies and children with TLD might consolidate and generalise better if the exposure duration was extended. However, keeping children engaged with a longer task and ensuring they paid attention throughout would be a challenge. This was one of the reasons why the current study presented each string of syllables four times only. Comparing to previous studies which found successful generalization in children with language impairments (Plante et al., 2014) and adults without LLD (Grunow et al., 2006), their exposure durations were obviously longer. Plante et al.'s (2014) “treatment sessions lasted $30 \mathrm{~min}$ and occurred daily for as many as 25 sessions" (p. 534) whereas Grunow et al. (2006) seemed to follow Gómez (2002) in presenting each string of syllables six times. Although Hsu et al. (2014) did not look at generalisation, they also followed Gómez's (2002) design and found successful non-adjacent dependency learning in adolescents without SLI but not in adolescents with SLI. Taking these findings together, one may suggest that a presentation of six times may be sufficient for typically developing individuals (including adults, adolescents and children) to learn and generalise non-adjacent dependencies whereas individuals with language impairments may need a more extended exposure of multiple presentations. However, the 18-month-old infants in Gómez (2002) could discriminate the trained and untrained non-adjacent dependencies with only one presentation in the training phase. Hence, how exposure duration may affect non-adjacent dependency learning across the lifespan in both typical and atypical development needs further investigation. If typically developing infants were more sensitive to statistical features despite low exposure whereas infants at risk of language disorders were not, then early identification and extended exposure throughout development may be beneficial for those who were in need to learn non-adjacent dependencies. 
Finally, variability needs to be considered. All of the studies discussed above have used 24 exemplars as high variability conditions in comparison with lower variability conditions (Gómez, 2002; Grunow et al., 2006; Hsu et al., 2014; Plante et al., 2014). They all suggested that high variability is the key for learning non-adjacent dependencies. Therefore, extended exposure needs to be paired with high variability in order to facilitate non-adjacent dependency learning in both typically and atypically developing individuals. High variability was also found to be important for atypically developing individuals in learning different grammatical structures (Plante et al., 2014; von Koss Torkildsen, Dailey, Aguilar, Gómez, \& Plante, 2013) and for typically developing adults in learning noun gender subcategories in a foreign language (Eidsvåg, Austad, Plante, \& Asbjørnsen, 2015). All these studies suggest a link between statistical learning and language acquisition and disorders although longitudinal evidence is still warranted.

In sum, the current study showed that Cantonese-speaking school-aged children with TLD were able to learn non-adjacent dependencies in non-inflectional artificial languages whereas children with SLI history were not. Taken together with findings from previous studies, this difficulty in individuals with atypical language development seems to be universal, regardless of age, diagnostic status, language and culture. Given that non-adjacent dependencies do not only occur in artificial and natural languages but also in other contexts (e.g., music, mathematics and science all involve abstracting long-distance relationships; Marcus, 2001), it is critical to investigate non-adjacent dependency learning in different contexts with relevance to other factors such as attention and exposure duration. Moreover, its longitudinal relationship with language acquisition and development in a much larger sample has to be determined in order to advance our understanding of language acquisition and language disorders and to provide implications for intervention and educational strategies. 


\section{Acknowledgements}

This study was partially supported by the University of Hong Kong through the Small

Project Funding awarded to Lai-Sang Iao. We thank the children, parents, and head teachers who made this research possible. Thanks also go to the anonymous reviewers for helpful suggestions. 


\section{References}

Arciuli, J., \& von Koss Torkildsen, J. (2012). Advancing our understanding of the link between statistical learning and language acquisition: The need for longitudinal data. Frontiers in psychology, 3, 324.

Chan, D. W., Ho, C. S.-H., Tsang, S.-M., Lee, S-H., \& Chung, K. K.-H. (2007). Prevalence, gender ratio, and gender differences in reading-related cognitive abilities among Chinese children with dyslexia in Hong Kong. Educational Studies, 33, 249-265.

Ching, Y. C. T. (1981). Communication of lexical tone patterns in Cantonese. Unpublished doctoral dissertation, University of London, London.

Cohen, J. (1992). A power primer. Psychological Bulletin, 112, 155-159.

Conway, C. M., \& Christiansen, M. H. (2006). Statistical learning within and between modalities pitting abstract against stimulus-specific representations. Psychological Science, 17, 905-912.

Ding, J. (2008). On the courses of the grammaticalization about Chinese temporal adverb "cengjing". Research in Theoretical Linguistics, 2, 15.

Eidsvåg, S. S., Austad, M., Plante, E., \& Asbjørnsen, A. E. (2015). Input Variability Facilitates Unguided Subcategory Learning in Adults. Journal of Speech, Language, and Hearing Research, 58, 826-839.

Evans, J. L., Saffran, J. R., \& Robe-Torres, K. (2009). Statistical learning in children with specific language impairment. Journal of Speech Language and Hearing Research, 52, 321-335.

Finneran, D. A., Francis, A. L., \& Leonard, L. B. (2009). Sustained attention in children with specific language impairment. Journal of Speech, Language, and Hearing Research, 52, 915-929.

Gebhart, A. L., Newport, E. L., \& Aslin, R. N. (2009). Statistical learning of adjacent and nonadjacent dependencies among nonlinguistic sounds. Psychonomic Bulletin \& Review, 16 , 486-490.

Gómez, R. L. (2002). Variability and detection of invariant structure. Psychological Science, 13, 431-436.

Green, D., \& Swets, J. (1974). Signal detection theory and psychophysics. Huntington, NY: Krieger. (Reprinted from original work published 1966, NY: Wiley)

Grunow, H., Spaulding, T. J., Gómez, R. L., \& Plante, E. (2006). The effects of variation on learning word order rules by adults with and without language-based learning disabilities. Journal of Communication Disorders, 39, 158-170.

Ho, C. S.-H., Chan, D.W.-O., Chung, K.K.-H., Tsang, S.-M., Lee, S.-H., \& Cheng, R. W.-Y. (2007). The Hong Kong Test of Specific Learning Difficulties in Reading and Writing for Primary School Students-Second Edition [HKT-P (II)]. Hong Kong: Hong Kong Specific Learning Difficulties Research Team. 
Hsu, H. J., Tomblin, J. B., \& Christiansen, M. H. (2014). Impaired statistical learning of non-adjacent dependencies in adolescents with specific language impairment. Frontiers in psychology, 5, 1-10.

Humanities Computing and Methodology Programme, Chinese University of Hong Kong. (2003). A Chinese character database: With word-formations phonologically disambiguated according to the Cantonese dialect. Retrieved on November 30, (2012). Web site: http://humanum.arts.cuhk.edu.hk/Lexis/lexi-can/

Kerkhoff, A., De Bree, E., De Klerk, M., \& Wijnen, F. (2013). Non-adjacent dependency learning in infants at familial risk of dyslexia. Journal of child language, 40, 11-28.

Leonard, L. B. (1998). Children with specific language impairment. Cambridge, MA: MIT Press.

Marcus, G. F. (2001). The algebraic mind: Integrating connectionism and cognitive science. Cambridge Mass: MIT Press.

Newport, E. L., \& Aslin, R. N. (2004). Learning at a distance: I. Statistical learning of nonadjacent dependencies. Cognitive Psychology, 48, 127-162.

Pacton, S., \& Perruchet, P. (2008). An attention-based associative account of adjacent and nonadjacent dependency learning. Journal of Experimental Psychology: Learning, Memory, and Cognition, 34, 80.

Plante, E., Ogilvie, T., Vance, R., Aguilar, J. M., Dailey, N. S., Meyers, C., ... \& Burton, R. (2014). Variability in the language input to children enhances learning in a treatment context. American Journal of Speech-Language Pathology, 23, 530-545.

Raven, J. C. (1986). Hong Kong Supplement Guide to the Standard Progressive Matrices. Hong Kong: HKSAR Government Education Department.

Romberg, A. R., \& Saffran, J. R. (2013). All together now: Concurrent learning of multiple structures in an artificial language. Cognitive science, 37, 1290-1320.

Saffran, J. R., Aslin, R. N., \& Newport, E. L. (1996). Statistical learning by 8-month-old infants. Science, 274, 1926-1928.

Spaulding, T. J., Plante, E., \& Vance, R. (2008). Sustained selective attention skills of preschool children with specific language impairment: Evidence for separate attentional capacities. Journal of Speech, Language, and Hearing Research, 51, 16-34.

T'sou, B., Lee, T., Tung, P., Man, Y., Chan, A., To, C. K. S., \& Chan, Y. (2006). Hong Kong Cantonese oral language assessment scale. Hong Kong: City University of Hong Kong.

von Koss Torkildsen, J., Dailey, N. S., Aguilar, J. M., Gómez, R., \& Plante, E. (2013). Exemplar variability facilitates rapid learning of an otherwise unlearnable grammar by individuals with language-based learning disability. Journal of Speech, Language, and Hearing Research, 56, 618-629.

Wong, A. M. Y., Ciocca, V., \& Yung, S. (2009). The perception of lexical tone contrasts in Cantonese children with and without specific language impairment (SLI). Journal of Speech, Language, and Hearing Research, 52, 1493-1509. 
Table 1

Demographic information and language mean scaled scores and standard deviations in the SLI-history and TLD Groups

\begin{tabular}{|c|c|c|c|c|c|c|}
\hline \multirow[t]{2}{*}{ Characteristic } & \multicolumn{3}{|c|}{$\begin{array}{c}\text { SLI-history }(n=16,10 \\
\text { males })\end{array}$} & \multicolumn{3}{|c|}{$\operatorname{TLD}(n=16,7$ males $)$} \\
\hline & $M$ & $S D$ & Min - Max & $M$ & $S D$ & Min - Max \\
\hline $\begin{array}{l}\text { Chronological age } \\
\text { (months) }\end{array}$ & 119 & 3.43 & $111-127$ & 116.25 & 8.06 & $100-126$ \\
\hline Age $5-6$ & & & & & & \\
\hline NVIQ & 109.25 & 8.60 & $91-127$ & 109 & 7.61 & $94-121$ \\
\hline Age $6-7$ & & & & & & \\
\hline HKCOLAS & & & & & & \\
\hline Vocabulary & $5.13 *$ & 1.71 & $2-8$ & 11.50 & 2.97 & $6-16$ \\
\hline Grammar & $4.81 *$ & 2.29 & $0-9$ & 11.12 & 2.50 & $6-15$ \\
\hline $\begin{array}{l}\text { Textual } \\
\text { Comprehension }\end{array}$ & $7.50 *$ & 1.71 & $5-11$ & 11.56 & 3 & $6-17$ \\
\hline $\begin{array}{l}\text { Narrative } \\
\text { Retelling }\end{array}$ & $5.75 *$ & 3.49 & $1-12$ & 11.38 & 2.92 & $7-17$ \\
\hline
\end{tabular}

Note. NVIQ $=$ Non-verbal intelligence quotient; HKCOLAS $=$ Hong Kong Cantonese Oral Language Assessment Scale; * Significant difference (t-test) from TLD at $p<.001$ 
Figure 1. Flow chart showing participant recruitment from a three-year longitudinal project to the current study.

Figure 2. Mean Scores for Correct Accepts and False Positives for Test and Generalization Items. 


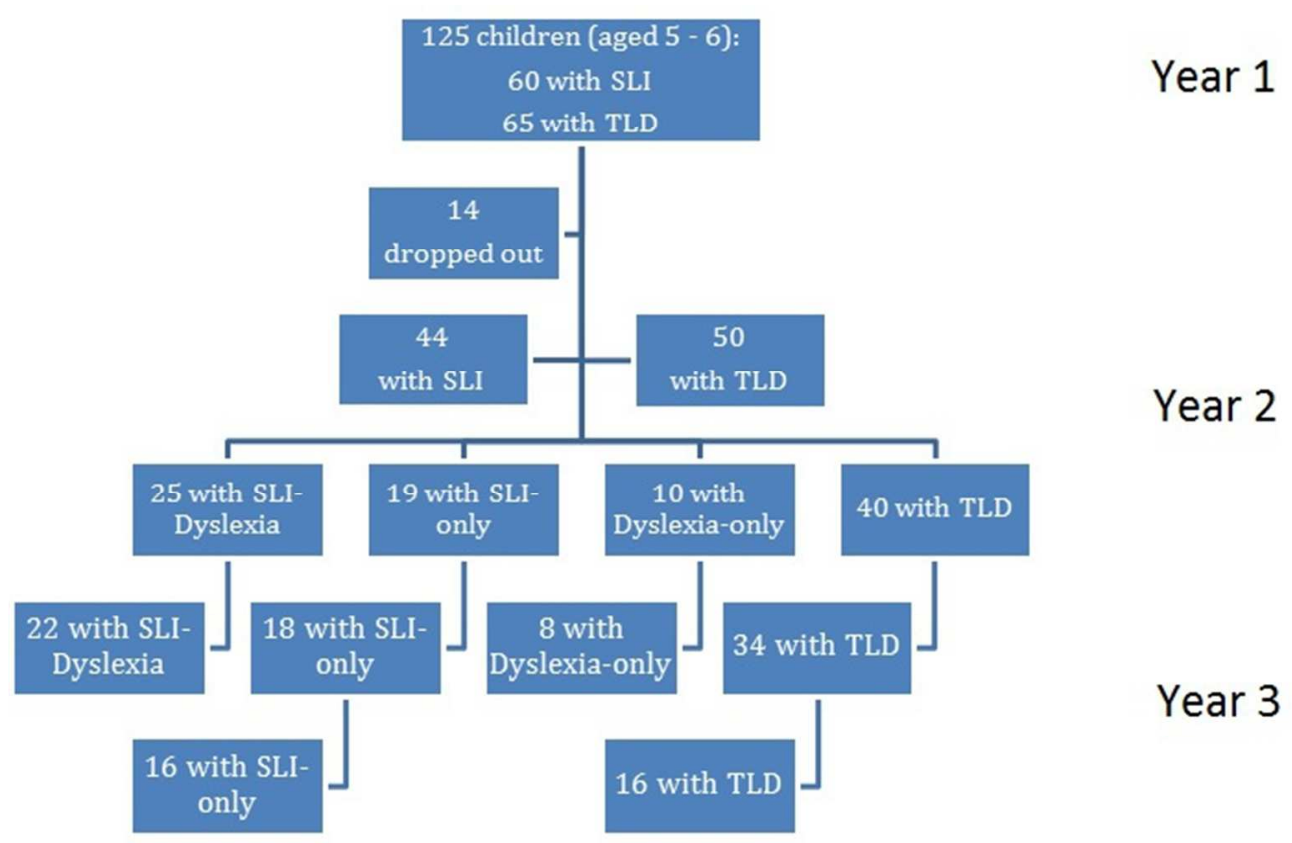

Flow chart showing participant recruitment from a three-year longitudinal project to the current study. $186 \times 122 \mathrm{~mm}(96 \times 96 \mathrm{DPI})$ 


\section{Mean Scores for Correct Accepts and False Positives for Test and Generalization Items.}

\section{$152 \times 98 \mathrm{~mm}(96 \times 96 \mathrm{DPI})$}




\section{Appendices}

\section{Appendix A}

Beginning, ending and intervening elements for both Language 1 and 2

The 2 beginning and 2 ending elements were in the form of consonant-vowel-consonant (CVC) pseudomorphemes: $/ \operatorname{tam}^{4} /, / f ø n^{4} /, / p \varepsilon k^{1} /$ and $/ h I p^{1} /$ respectively. The intervening elements were in the form of $\mathrm{CV}+\mathrm{CVC}$ pseudomorphemes as follows:
$X_{1} \quad / \mathrm{ja}^{1} \mathrm{p}^{\mathrm{h}} \mathrm{et}^{4} /$
$X_{10} \quad / \mathrm{p}^{\mathrm{h}} \mathfrak{}^{1} \mathrm{k}^{\mathrm{h}} \mathrm{Im}^{1} /$
$X_{19} \quad / \operatorname{tsu}^{4} \operatorname{tsin}^{1} /$
$X_{2} \quad / \mathrm{j}^{1} \mathrm{k}^{\mathrm{h}} \mathrm{en}^{4} /$
$X_{11} \quad / \mathrm{fo}^{1} \ln ^{1} /$
$X_{20} \quad / \mathrm{fy}^{4} \mathrm{mcp}^{1} /$
$X_{3} \quad / \mathrm{fI}^{1} \mathrm{pem}^{4} /$
$X_{12} \quad / \mathrm{k}^{1} \mathrm{jip}^{1} /$
$X_{21} \quad / \mathrm{lu}^{4} \operatorname{sip}^{1} /$
$X_{4} \quad / \mathrm{tr}^{1}{\mathrm{j} \varepsilon \mathrm{m}^{4} /}^{4}$
$X_{13} \quad / \mathrm{p \varepsilon}^{4} \operatorname{tøn}^{4} /$
$X_{22} \quad / \mathrm{hu}^{4} \mathrm{kot}^{1} /$
$X_{5} \quad / \mathrm{t}^{\mathrm{h}} \mathrm{I}^{1} \operatorname{pan}^{4} /$
$X_{14} \quad / \mathrm{ta}^{4} \mathrm{fem}^{4} /$
$X_{23} \quad /$ py $^{4}$ høn $^{1} /$
$X_{6} \quad / \mathrm{k}_{\mathrm{I}}^{\mathrm{h}^{1}} \mathrm{ts}^{\mathrm{h}} \mathrm{ap}^{4} /$
$X_{15} / \mathrm{tsa}^{4} \mathrm{kan}^{4} /$
$X_{24} \quad / \mathrm{my}^{4} \operatorname{lap}^{1} /$
$X_{7} \quad / \mathrm{wI}^{1} \mathrm{lak}^{1} /$
$X_{16} / \mathrm{ko}^{4} \mathrm{sam}^{4} /$
$X_{8} \quad / \mathrm{p}^{1} \mathrm{f} \mathrm{k}^{1} /$
$X_{17} \quad / \mathrm{po}^{4} \mathrm{j}^{\mathrm{k}} \mathrm{m}^{4} /$
$X_{9} \quad / \mathrm{m}^{1} \mathrm{pem}^{1} /$
$X_{18} / \mathrm{fo}^{4} \mathrm{tIm}^{4} /$ 


\section{Appendix B}

Test strings used in testing phase

\begin{tabular}{|c|c|c|}
\hline Item & Language 1 & Language 2 \\
\hline \multirow[t]{6}{*}{ Test } & $/ \operatorname{tam}^{4} \mathrm{ja}^{1} \mathrm{p}^{\mathrm{h}} \mathrm{et}^{4} \mathrm{hrp}^{1} /$ & $/ \operatorname{tam}^{4} \mathrm{ja}^{\mathrm{l}} \mathrm{p}^{\mathrm{h}} \mathrm{et}^{4} \mathrm{p} \mathrm{k}^{\mathrm{I}} /$ \\
\hline & $/ \operatorname{tam}^{4} \mathrm{wI}^{1} \mathrm{lak}^{1} \mathrm{hip}^{1} /$ & $/ \operatorname{tam}^{4} \mathrm{wI}^{1} \mathrm{lak}^{1} \mathrm{pek}^{1} /$ \\
\hline & $/ \operatorname{tam}^{4} \mathrm{hu}^{4} \mathrm{kst}^{1} \mathrm{hrp}^{1} /$ & $/ \operatorname{tam}^{4} \mathrm{hu}^{4} \mathrm{kst}^{1} \mathrm{p} \mathrm{k}^{1} /$ \\
\hline & $/ \mathrm{føn}^{4} \mathrm{ja}^{1} \mathrm{p}^{\mathrm{h}} \mathrm{pt}^{4} \mathrm{p} \mathrm{k}^{1} /$ & $/ \mathrm{føn}^{4} \mathrm{ja}^{1} \mathrm{p}^{\mathrm{h}} \mathrm{et}^{4} \mathrm{hrp} \mathrm{p}^{1} /$ \\
\hline & $/ \mathrm{føn}^{4} \mathrm{wI}^{1} \mathrm{lak}^{1} \mathrm{p} \varepsilon \mathrm{k}^{1} /$ & $/$ føn $^{4} \mathrm{wI}^{1} \mathrm{lak}^{1} \mathrm{hip}^{1} /$ \\
\hline & $/ \mathrm{føn}^{4} h \mathrm{u}^{4} \mathrm{kot}^{1} \mathrm{p} \varepsilon \mathrm{k}^{1} /$ & $/$ føn ${ }^{4} h u^{4} \mathrm{kot}^{1} \mathrm{hrp}{ }^{1} /$ \\
\hline \multirow[t]{6}{*}{ Generalization } & $/ \operatorname{tam}^{4} w^{1} \mathrm{fok}^{1} \mathrm{hrp}^{1} /$ & $/ \operatorname{tam}^{4} w^{1}$ fok ${ }^{1} \mathrm{pck}^{1 /}$ \\
\hline & $/$ tam $^{4}$ hy $^{4}$ fak $^{1}$ hip $^{1} /$ & $/ \operatorname{tam}^{4} \mathrm{hy}^{4} \mathrm{fak}^{1} \mathrm{p} \mathrm{k}^{1} /$ \\
\hline & $/ \operatorname{tam}^{4} \mathrm{k}^{\mathrm{h}} \mathrm{i}^{1} \operatorname{len}^{4} \mathrm{hrp}^{1} /$ & $/ \operatorname{tam}^{4} \mathrm{k}^{\mathrm{h}^{1}}{ }^{1} \operatorname{len}^{4} \mathrm{p} \mathrm{k}^{1} /$ \\
\hline & $/ \mathrm{f} ø \mathrm{n}^{4} \mathrm{w}^{1} \mathrm{f}^{1} \mathrm{k}^{1} \mathrm{p} \varepsilon \mathrm{k}^{1 /}$ & /føn ${ }^{4} w^{1}$ fok $^{1} h ı p^{1} /$ \\
\hline & $/ \mathrm{føn}^{4} \mathrm{hy}^{4} \mathrm{fak}^{1} \mathrm{p} \varepsilon \mathrm{k}^{1} /$ & $/$ føn $^{4} \mathrm{hy}^{4} \mathrm{fak}^{1} \mathrm{hup}^{1} /$ \\
\hline & $/ \mathrm{føn}^{4} \mathrm{k}^{\mathrm{h}} \mathrm{i}^{1} \operatorname{len}^{4} \mathrm{p} \varepsilon \mathrm{k}^{1} /$ & $/ \mathrm{føn}^{4} \mathrm{k}^{\mathrm{h}} \mathrm{i}^{1} \operatorname{len}^{4} \mathrm{p} \varepsilon \mathrm{k}^{1} /$ \\
\hline
\end{tabular}

